\title{
Commentary: Where have all the lymph nodes gone?
}

\author{
Benjamin D. Kozower, MD, MPH
}

\author{
From the Washington University in St Louis, St Louis, Mo. \\ Disclosures: Author has nothing to disclose with regard to commercial support. \\ Received for publication Feb 4, 2019; accepted for publication Feb 5, 2019; available ahead of print March 20, \\ 2019. \\ Address for reprints: Benjamin D. Kozower, MD, MPH, 660 S. Euclid Ave, Campus Box 8234, St Louis, MO \\ 63110 (E-mail: kozowerb@wustl.edu). \\ J Thorac Cardiovasc Surg 2019;157:2468 \\ $0022-5223 / \$ 36.00$ \\ Copyright (C) 2019 by The American Association for Thoracic Surgery \\ https://doi.org/10.1016/j.jtcvs.2019.02.020
}

Dr Stiles and colleagues ${ }^{1}$ have conducted another outstanding study demonstrating that the majority of patients undergoing sublobar resection receive an inadequate lymph node sampling/dissection. Their results using Surveillance, Epidemiology, and End Results Medicare data demonstrate that $46.9 \%$ of patients with T1N0 ( $<2-\mathrm{cm}$ cancers) treated with sublobar resection have no lymph nodes sampled. Unfortunately, these results are similar to a recent publication of ours using the National Cancer Database demonstrating that the median lymph node count for sublobar resection is 1 node. ${ }^{2}$ The article by Stiles and colleagues ${ }^{1}$ also demonstrates that sublobar resection is associated with a lower overall survival and cancer-specific survival compared with lobectomy. In an interesting analysis, when the sublobar group was limited to the $8 \%$ of adequately staged patients with at least 9 lymph nodes, there were no differences in overall or cancer-specific survival.

There are 2 limitations noted by the authors that influence their results. ${ }^{1}$ First, the present study uses pathologic staging rather than clinical staging. This is an important distinction because the lobectomy group was more likely to have lymph nodes sampled. Therefore, patients receiving lobectomy more likely will be upstaged, removed from the pathologic stage I sample used in the study, and accentuate the survival differences between the surgical groups. Second, the majority of the sublobar group consists of patients treated with wedge resection $(81 \%)$. In a recent analysis of Society of Thoracic Surgeons data linked with Medicare to obtain 5-year survival, the survival for patients with stage I treated with segmentectomy equaled that of lobectomy. ${ }^{3}$ This recent study provides a caution flag for grouping wedge resection and anatomic segmentectomy together into a sublobar group. They are very different procedures, and when routinely performed in the United States result in very different lymph node evaluations.

The article by Stiles and colleagues ${ }^{1}$ highlights a concerning issue with lung cancer resection in the United States. Approximately one-third of patients with surgically resected stage I lung cancer receive sublobar resection, and



the majority of these patients do not receive an adequate lymph node evaluation/dissection. This is critical to keep in mind when the results of prospective randomized controlled trials comparing lobectomy with sublobar resection are released in the near future. Those prospective trials are not pragmatic and mandated a thorough lymph node evaluation before randomization. Therefore, it will be difficult to generalize the results of those trials to routine clinical practice in the United States when half of patients treated with sublobar resection have no lymph nodes evaluated. This is a critical issue that needs to be addressed by the thoracic surgery community. The American College of Surgeons Commission on Cancer recognizes this issue, and a task force is working on implementing a quality of care measure for lymph node evaluation during lung cancer resection. $^{4}$

\section{References}

1. Stiles BM, Mao J, Harrison S, Lee B, Port JL, Sedrakyan A, et al. Extent of lymphadenectomy is associated with oncologic efficacy of sublobar resection for lung cancer 2 cm. J Thorac Cardiovasc Surg. 2019;157:2454-65.e1.

2. Subramanian M, McMurry T, Meyers BF, Puri V, Kozower BD. Long-term results for clinical stage IA lung cancer: comparing lobectomy and sublobar resection. Ann Thorac Surg. 2018;106:375-81.

3. Onaitis M, Fumary AP, Kosinski AS, Feng L, Boffa DJ, Tong BC, et al. Equivalent survival between lobectomy and segmentectomy for lung cancer: an analysis of elderly clinical stage IA patients in the STS general thoracic surgery database. Ann Thorac Surg. 2019 [In press].

4. The American College of Surgeons-Commission on cancer quality of care measures. Available at: https://www.facs.org/quality-programs/cancer/ncdb/quality measures. Accessed February 4, 2019. 